\title{
Práticas integrativas e complementares, transtornos mentais e satisfação com a vida
}

\section{entre universitários}

Integrative and complementary practices, mental disorders and life satisfaction among university students

Prácticas integrativas y complementarias, trastornos mentales y satisfacción con la vida en los estudiantes

universitarios

Recebido: 14/06/2021 | Revisado: 22/06/2021 | Aceito: 24/06/2021 | Publicado: 12/07/2021

\author{
Vanessa Soares Alves Nascimento \\ ORCID: https://orcid.org/0000-0001-7581-7283 \\ Universidade Federal de Sergipe, Brasil \\ E-mail: vansoares.12@gmail.com \\ Anne Manuelle dos Santos \\ ORCID: https://orcid.org/0000-0002-2049-8593 \\ Universidade Federal de Sergipe, Brasil \\ E-mail: annemanuelle63@gmail.com \\ Shirley Verônica Melo Almeida Lima \\ ORCID: https://orcid.org/0000-0002-9062-0742 \\ Universidade Federal de Sergipe, Brasil \\ E-mail: shirleymelo.lima@gmail.com \\ Glebson Moura Silva \\ ORCID: https://orcid.org/0000-0002-4977-2787 \\ Universidade Federal de Sergipe, Brasil \\ E-mail: glebsonmoura@yahoo.com.br \\ Carla Kalline Alves Cartaxo Freitas \\ ORCID: https://orcid.org/0000-0001-7604-9132 \\ Universidade Federal de Sergipe, Brasil \\ E-mail: carlakalline@gmail.com \\ Andreia Freire de Menezes \\ ORCID: https://orcid.org/0000-0002-2972-8236 \\ Universidade Federal de Sergipe, Brasil \\ E-mail: deiamenezes@hotmail.com
}

\section{Resumo}

Objetivo: identificar as relações entre satisfação com a vida, sintomas de ansiedade e depressão e, práticas integrativas e complementares em estudantes universitários da área de saúde. Metodologia: pesquisa descritiva quantitativa com discentes da Universidade Federal de Sergipe. O período de coleta ocorreu entre os meses de agosto de 2018 e novembro de 2019. Associações entre as variáveis categóricas foram realizadas pelo teste qui-quadrado de pearson. Resultados: associação significativa foi encontrada entre os sintomas de ansiedade e o período em que os alunos se encontram na grade acadêmica, módulo perdido e queixa principal. Os sintomas de depressão apresentaram associação com o grupo etário, módulo perdido e satisfação com o curso. Conclusão: Existe dependência condicional e a relação da satisfação com a vida entre idade, curso, satisfação com o curso e satisfação com o método é influenciada pela condição de frequentar ou não a sala de cuidados.

Palavras-chave: Ansiedade; Depressão; Práticas Integrativas; Satisfação com a vida; Estudantes universitários.

\begin{abstract}
Objective: to identify the relationships between satisfaction with life, symptoms of anxiety and depression, and integrative and complementary practices in university students in the health area. Methodology: quantitative descriptive research with students from the Federal University of Sergipe. The collection period took place between the months of August 2018 and November 2019. Associations between categorical variables were performed using Pearson's chisquare test. Results: a significant association was found between anxiety symptoms and the period in which students are in the academic grade, lost module and main complaint. Depression symptoms were associated with age group, lost module and satisfaction with the course. Conclusion: There is conditional dependence and the relationship of satisfaction with life between age, course, satisfaction with the course and satisfaction with the method is influenced by the condition of attending or not the care room.
\end{abstract}

Keywords: Anxiety; Depression; Integrative practices; Satisfaction with life; University students. 


\begin{abstract}
Resumen
Objetivo: identificar las relaciones entre satisfacción con la vida, síntomas de ansiedad y depresión y prácticas integradoras y complementarias en estudiantes universitarios del área de la salud. Metodología: investigación descriptiva cuantitativa con estudiantes de la Universidad Federal de Sergipe. El período de recolección se llevó a cabo entre los meses de agosto de 2018 y noviembre de 2019. Las asociaciones entre variables categóricas se realizaron mediante la prueba de chi-cuadrado de Pearson. Resultados: se encontró asociación significativa entre los síntomas de ansiedad y el período en el que los estudiantes se encuentran en el grado académico, módulo perdido y queja principal. Los síntomas de depresión se asociaron con el grupo de edad, el módulo perdido y la satisfacción del curso. Conclusión: Existe dependencia condicional y la relación de satisfacción con la vida entre edad, curso, satisfacción con el curso y satisfacción con el método está influenciada por la condición de asistir o no a la sala de cuidados.
\end{abstract}

Palabras clave: Ansiedad; Depresión; Prácticas integradoras; Satisfacción con la vida; Estudiantes universitarios.

\title{
1. Introdução
}

A vida universitária proporciona o contato com novos conhecimentos, valores e crenças, questionamentos e experiências acadêmicas e sociais que podem propiciar aos estudantes um amadurecimento pessoal e que certamente irão repercutir sobre a profissão escolhida (Carleto et.al, 2018). As mudanças geradas na vida do estudante universitário geralmente são responsáveis por diversas situações estressantes que podem aumentar progressivamente, pois, a cada etapa do curso surgem exigências que requererão o desenvolvimento de habilidades e/ou competências por parte dos estudantes (Cestari, 2017).

Nos últimos anos, a saúde mental de estudantes universitários tornou-se foco de atenção de especialistas da área de saúde e da sociedade em geral, pois nota-se a ocorrência de situações geradoras de sofrimento psíquico e suas manifestações sintomáticas como a ansiedade e depressão que, podem ter interferir na relação com os pacientes (Vasconcelos et.al, 2015).

A prevalência de depressão na população mundial é de cerca de 4,4\% e a de ansiedade 3,6\%. Tem-se ainda que a depressão é o maior contribuidor do YLD (years lived with disability), correspondendo a 7,5\% de todos os anos vividos com incapacidade em 2015 e a ansiedade está em sexto lugar com 3,4\%. Estudos têm indicado que a Ansiedade e a Depressão resultam da interação de diferentes fatores sociais, psicológicos e biológicos e podem trazer severos prejuízos, individuais, sociais e econômicos (Who, 2017).

Para Kumar et al. (2016), o sofrimento mental é um importante problema de saúde pública em todo o mundo e tem afetado cada vez mais a população mais jovem. Embora saibamos que os problemas de saúde mental afetam a sociedade como um todo, universitários têm alta prevalência de doenças psicológicas em comparação com a população em geral. Fatores decorrentes da experiência acadêmica podem levar ao desenvolvimento de problemas psicológicos e afetam negativamente o desempenho acadêmico. Estudos nacionais e internacionais indicam alto índice de problemas psicológicos como depressão, ansiedade e estresse nos alunos.

Nesse sentido, tem-se a importância de se oferecer possibilidades de intervenções terapêuticas utilizando de ferramentas públicas disponíveis a fim de promover uma melhor qualidade de vida dos estudantes universitários. Uma importante ferramenta é a Política Nacional de Práticas Integrativas e Complementares (Pics) que foi estabelecida no Sistema Único de Saúde (SUS) em 2006. As Pics são tratamentos que utilizam recursos terapêuticos baseados em conhecimentos tradicionais, voltados para prevenir diversas doenças e em alguns casos, indicadas também como tratamentos paliativos em algumas doenças crônicas. (Brasil, 2018).

Para Souza e Barros (2018), as Pics podem oferecer soluções alternativas ao sofrimento mental dos universitários, bem como à valorização do indivíduo como centro do cuidado, à busca por meios terapêuticos mais simples, menos dependentes de tecnologia científica dura, mais econômicos e com igual ou maior eficácia nas situações comuns de adoecimento.

Assim, para uma melhor qualidade de vida e aumentar a probabilidade de se sentir satisfeito com a mesma, torna-se relevante a capacidade de identificar e controlar os sintomas da ansiedade e da depressão, sobretudo em estudantes universitários.

A Satisfação com a vida, componente cognitivo do bem estar subjetivo, é um construto que se refere à avaliação 
cognitiva que o indivíduo faz sobre sua qualidade de vida geral ou relativa à domínios tais como trabalho, lazer, saúde, finanças, dentre outos. Essa avaliação é feita a partir da comparação entre as circunstâncias de vida do indivíduo e um padrão que cada indivíduo determina para si (Hutz, Zanon \& Bardagi, 2014).

Estudos realizados em universidades brasileiras também desenvolveram temáticas com estudantes, como ansiedade e depressão (Costa et al, 2020), práticas integrativas e complementares (Santos et al, 2019) e satisfação com a vida (Silva, Heleno 2012; Suehiro, Andrade, 2018), porém com distintos olhares e perspectivas. A Universidade Federal de Sergipe, campus Lagarto, é caracterizada por um cenário diferente por desenvolver uma metodologia de ensino aprendizagem ativa a qual ocoore em poucas instituições de ensino superior no Brasil. A presente pesquisa pode contribuir para o processo de resgate da concepção de saúde nesta população além de identificar a ocorrência da satisfação com a vida e sintomas de ansiedade e depressão em estudantes universitários da área de saúde.

Nesse sentido, este trabalho objetivou analisar a relação entre sintomas de ansiedade e depressão, práticas integrativas e, satisfação com a vida entre estudantes universitários, além de dimensionar a realidade local no contexto de saúde mental desta população que poderá auxiliar na elaboração de estratégias facilitadoras do desenvolvimento de ações de saúde destes futuros profissionais.

\section{Metodologia}

Trata-se de uma pesquisa descritiva quantitativa com discentes da Universidade Federal de Sergipe, campus Prof. Antônio Garcia Filho, localizada na cidade de Lagarto. O Campus oferece oito cursos de graduação na área de saúde, estruturados a partir da Aprendizagem Baseada em Problemas (ABP) e Problematização. Além disso, consideramos que, por ser uma Universidade localizada em uma cidade do interior do estado de Sergipe, muitos universitários vêm de fora e se distanciam da família, trazendo consigo vulnerabilidade emocional e física o que pode resultar em ansiedade e/ou depressão.

Os ambientes de coleta dos dados foram a sala de cuidados, laboratórios, centro de convivência (espaço de socialização entre os estudantes) e salas de aula do Campus. A sala de cuidados trata-se de um espaço onde são oferecidas três práticas integrativas e complementares aos estudantes que acessam o serviço: o reiki, a massoterapia e a auriculoterapia. A equipe de cuidadores é composta por alunos dos cursos de extensão oferecidos pelo Movimento Popular de Saúde (MOPS) em parceria com a UFS.

A pesquisa ocorreu entre os meses de agosto de 2018 e novembro de 2019, através de três instrumentos: uma ficha de caracterização do perfil sociodemográfico e clínico e, duas escalas - a Escala Hospitalar de Ansiedade e Depressão (Zigmond \& Snaith, 1983), traduzida e validada no Brasil (Botega et al., 1995) que contém 14 questões do tipo múltipla escolha. Compõe-se de duas subescalas, para ansiedade e depressão, com sete itens cada. A pontuação global em cada subescala vai de 0 a 21 e a Escala de Satisfação com a Vida (ESV), elaborada por Diener, Emmons, Larsen e Griffin (1985), composta por cinco itens do tipo Likert de 7 pontos, em que 1 significa discordo plenamente e 7 concordo plenamente. Após o somatório da cada item, os pontos de corte usados como benchmarks pela escala foram aplicados e, posteriormente categorizados em satisfeitos e não satisfeitos com a vida.

Os dados foram tabulados no programa Excel e as informações submetidas à análise através do programa Statistical Package for Social Sciences (SPSS) 22.0 para Windows (SPSS Inc., Chicago, Illinois), sendo considerado como significância estatística um $\mathrm{p}<0,05$ para rejeição da hipótese nula. Associações entre as variáveis categóricas foram realizadas pelo teste quiquadrado de pearson. A fim de explorar as práticas integrativas e complementares realizadas com os estudantes universitários, foi construído um modelo de regressão logística múltipla (ajustado pelo teste Hosmer-Lemeshow e curva ROC) considerando os alunos que frequentavam ou não a sala de cuidados e os aspectos sociodemográficos, ansiedade e depressão e, de satisfação com a vida. Para considerar a independência condicional, os dados foram analisados pelo teste de Mantel-Haenszel e por meio 
do modelo log-linear.

Todos os participantes ficaram cientes em relação ao objetivo da pesquisa, além de terem participação voluntária sendo garantido anonimato. A pesquisa foi aprovada pelo comitê de ética e pesquisa da Universidade Federal de Sergipe sob o parecer: 2.771.738 e está de acordo com os princípios éticos de não maleficência, beneficência, justiça e autonomia.

\section{Resultados}

O estudo obteve uma amostra de 305 alunos de graduação da área de saúde considerando a participação (170) ou não (135) na sala de Cuidados Integrativos. Os alunos possuíam predominantemente entre 20 e 29 anos de idade, a maioria do sexo feminino $(71,8 \% / 219)$, solteiro(a) $(97 \% / 296)$, sem trabalho formal $(82,3 \% / 251)$, vivia com a família $(58,3 \% / 178)$.

A maioria dos estudantes se encontrava nos primeiros $50 \%$ da graduação $(93,4 \% / 285)$. No tocante às características relacionadas ao curso, apenas 13,8\% relataram a perda de algum módulo durante a graduação, e a maioria relatou satisfação com o curso $(89,2 \% / 272)$ e com o método de ensino adotado pela Universidade $(77,3 \% / 236)$.

Entre os alunos que frequentaram a sala de cuidados, associação significativa foi encontrada entre os sintomas de ansiedade e o período em que os alunos se encontram na grade acadêmica $(p<0,038)$, módulo perdido $(p<0,020)$ e queixa principal $(\mathrm{p}<0,025)$. Os sintomas de depressão apresentaram associação com o grupo etário $(\mathrm{p}<0,003)$, módulo perdido $(p<0,003)$ e satisfação com o curso $(p<0,024)$. (Tabela 1) 


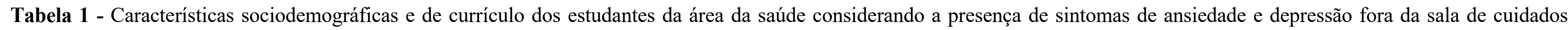
integrativos, Lagarto, Sergipe, 2020.

\begin{tabular}{|c|c|c|c|c|c|c|c|c|c|c|}
\hline \multirow{3}{*}{ Características sociodemográficas } & \multicolumn{10}{|c|}{ Dentro da Sala de Cuidados Integrativos } \\
\hline & \multicolumn{5}{|c|}{ Ansiedade $(n=170)$} & \multicolumn{5}{|c|}{ Depressão (n=170) } \\
\hline & $\begin{array}{l}\text { Normal } \\
\text { n }(\%)\end{array}$ & $\begin{array}{l}\text { Discreto } \\
\mathrm{n}(\%)\end{array}$ & $\begin{array}{l}\text { Moderado } \\
\mathrm{n}(\%)\end{array}$ & $\begin{array}{l}\text { Severo } \\
\mathrm{n}(\%)\end{array}$ & p-valor* & $\begin{array}{l}\text { Normal } \\
\mathrm{n}(\%)\end{array}$ & $\begin{array}{l}\text { Discreto } \\
\mathrm{n}(\%)\end{array}$ & $\begin{array}{l}\text { Moderado } \\
\mathrm{n}(\%)\end{array}$ & $\begin{array}{l}\text { Severo } \\
\mathrm{n}(\%)\end{array}$ & p-valor* \\
\hline \multicolumn{11}{|l|}{ Sexo } \\
\hline Masculino & $15(30)$ & $14(28)$ & $16(32)$ & $5(10)$ & 0,385 & $33(66)$ & $12(24)$ & $4(8)$ & $1(2)$ & 0,413 \\
\hline Feminino & $22(18,3)$ & $35(29,2)$ & $46(38,3)$ & $17(14,2)$ & & $74(61,7)$ & $36(30)$ & $10(8,3)$ & $0(0)$ & \\
\hline $\begin{array}{l}\text { Período } \\
<50 \% \\
>50 \%\end{array}$ & $\begin{array}{l}31(19,5) \\
6(54,5)\end{array}$ & $\begin{array}{l}47(29,6) \\
2(18,2)\end{array}$ & $\begin{array}{l}59(37,1) \\
3(27,3)\end{array}$ & $\begin{array}{l}22(13,8) \\
0(0)\end{array}$ & 0,038 & $\begin{array}{l}96(60,4) \\
11(100)\end{array}$ & $\begin{array}{l}48(30,2) \\
0(0)\end{array}$ & $\begin{array}{l}14(8,8) \\
0(0)\end{array}$ & $\begin{array}{l}1(0,6) \\
0(0)\end{array}$ & 0,091 \\
\hline \multicolumn{11}{|l|}{ Módulo perdido } \\
\hline $\begin{array}{l}\text { Sim } \\
\text { Não }\end{array}$ & $\begin{array}{l}3(10,7) \\
34(23,9)\end{array}$ & $\begin{array}{l}4(14,3) \\
45(31,7)\end{array}$ & $\begin{array}{l}14(50) \\
48(33,8)\end{array}$ & $\begin{array}{l}7(25) \\
15(10,6)\end{array}$ & 0,020 & $\begin{array}{l}10(35,7) \\
97(68,3)\end{array}$ & $\begin{array}{l}13(46,4) \\
35(24,6)\end{array}$ & $\begin{array}{l}4(14,3) \\
10(7)\end{array}$ & $\begin{array}{l}1(3,6) \\
0(0)\end{array}$ & 0,003 \\
\hline $\begin{array}{l}\text { Satisfeito curso } \\
\text { Sim } \\
\text { Não }\end{array}$ & $\begin{array}{l}34(22,8) \\
3(17,6)\end{array}$ & $\begin{array}{l}45(30,2) \\
4(23,5)\end{array}$ & $\begin{array}{l}54(36,2) \\
6(35,3)\end{array}$ & $\begin{array}{l}16(10,7) \\
4(23,5)\end{array}$ & 0,527 & $\begin{array}{l}98(65,8) \\
7(41,2)\end{array}$ & $\begin{array}{l}39(26,2) \\
7(41,2)\end{array}$ & $\begin{array}{l}12(8,1) \\
2(11,8)\end{array}$ & $\begin{array}{l}0(0) \\
1(5,9)\end{array}$ & 0,024 \\
\hline \multicolumn{11}{|l|}{ Queixa Principal } \\
\hline Ansiedade & $4(8,7)$ & $10(21,7)$ & $23(50)$ & $9(19,6)$ & & $28(60,9)$ & $14(30,4)$ & $4(8,7)$ & $0(0)$ & \\
\hline Cefaleia & $3(27,3)$ & $2(18,2)$ & $4(36)$ & $2(18,2)$ & & $5(45,5)$ & $5(45,5)$ & $1(9,1)$ & $0(0)$ & \\
\hline Dor no corpo & $13(31)$ & $13(31)$ & $13(31)$ & $3(7,1)$ & & $32(76,2)$ & $7(16,7)$ & $3(7,1)$ & $0(0)$ & \\
\hline Estresse & $4(12,9)$ & $9(29)$ & $13(41,9)$ & $5(16,1)$ & 0,025 & $15(48,4)$ & $12(38,7)$ & $4(12,9)$ & $0(0)$ & 0,101 \\
\hline Fadiga mental & $1(14,3)$ & $5(71,4)$ & $1(14,3)$ & $0(0)$ & & $6(85,7)$ & $1(14,3)$ & $0(0)$ & $0(0)$ & \\
\hline Fadiga muscular & $8(53,3)$ & $3(20)$ & $3(20)$ & $1(6,7)$ & & $9(60)$ & $5(33,3)$ & $1(6,7)$ & $0(0)$ & \\
\hline Sono alterado & $1(12,5)$ & $2(25)$ & $3(37,5)$ & $2(25)$ & & $3(37,5)$ & $3(37,5)$ & $1(12,5)$ & $1(12,5)$ & \\
\hline Nenhuma & $4(40)$ & $3(60)$ & $0(0)$ & $0(0)$ & & $5(100)$ & $0(0)$ & $0(0)$ & $0(0)$ & \\
\hline
\end{tabular}

* Qui quadrado de Pearson. Fonte: Autores (2021). 
Achados fora da sala de cuidados revelaram presença de associações entre os sintomas de ansiedade e sexo ( $p<0,008$ ), condição de viver com a família ( $<<0,052$ ), módulo perdido

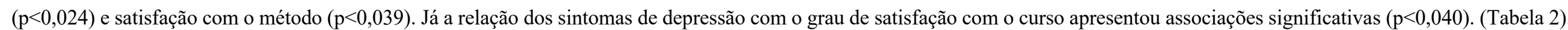

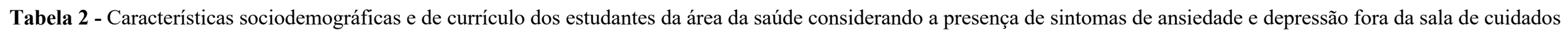
integrativos, Lagarto, Sergipe, 2020.

\begin{tabular}{|c|c|c|c|c|c|c|c|c|c|c|}
\hline \multirow{3}{*}{ Características sociodemográficas } & \multicolumn{10}{|c|}{ Fora da Sala de Cuidados Integrativos } \\
\hline & \multicolumn{5}{|c|}{ Ansiedade (n=135) } & \multicolumn{5}{|c|}{ Depressão (n=135) } \\
\hline & $\begin{array}{l}\text { Normal } \\
\mathrm{n}(\%)\end{array}$ & $\begin{array}{l}\text { Discreto } \\
\mathrm{n}(\%)\end{array}$ & $\begin{array}{l}\text { Moderado } \\
\mathrm{n}(\%)\end{array}$ & $\begin{array}{l}\text { Severo } \\
\mathrm{n}(\%)\end{array}$ & p-valor* & $\begin{array}{l}\text { Normal } \\
\mathrm{n}(\%)\end{array}$ & $\begin{array}{l}\text { Discreto } \\
\mathrm{n}(\%)\end{array}$ & $\begin{array}{l}\text { Moderado } \\
\mathrm{n}(\%)\end{array}$ & $\begin{array}{l}\text { Severo } \\
\mathrm{n}(\%)\end{array}$ & p-valor* \\
\hline Sexo & & & & & 0008 & & & & & 0864 \\
\hline Masculino & $16(44,4)$ & $8(22,2)$ & $9(25)$ & $3(8,3)$ & 0,008 & $21(58,3)$ & $9(25)$ & $5(13,9)$ & $1(2,8)$ & 0,004 \\
\hline Feminino & $17(17,2)$ & $23(23,2)$ & $42(42,4)$ & $17(17,2)$ & & $64(64,6)$ & $24(24,2)$ & $9(9,1)$ & $2(2)$ & \\
\hline Vive família & & & & & 0025 & & & & & 0820 \\
\hline Sim & $15(17,6)$ & $22(25,9)$ & $31(36,5)$ & $17(20)$ & $0,0<5$ & $51(60)$ & $23(27,1)$ & $9(10,6)$ & $2(2,4)$ & $0,8<9$ \\
\hline Não & $17(36,2)$ & $8(17)$ & $19(40,4)$ & $3(6,4)$ & & $32(68,1)$ & $9(19,1)$ & $5(10,6)$ & $1(2,1)$ & \\
\hline Módulo perdido & & & & & 0024 & & & & & 0098 \\
\hline $\operatorname{Sim}$ & $0(0)$ & $6(42,9)$ & $4(28,6)$ & $4(28,6)$ & $0,0<4$ & $5(35,7)$ & $5(35,7)$ & $3(21,4)$ & $1(7,1)$ & 0,090 \\
\hline Não & $33(27,5)$ & $24(20)$ & $47(39,2)$ & $16(13,3)$ & & $79(65,8)$ & $28(23,3)$ & $11(9,2)$ & $2(1,7)$ & \\
\hline Satisfeito curso & & & & & 0098 & & & & & 0,40 \\
\hline Sim & $31(25,2)$ & $29(23,6)$ & $46(37,4)$ & $17(13,8)$ & 0,098 & $80(65)$ & $29(23,6)$ & $11(8,9)$ & $3(2,4)$ & 0,040 \\
\hline Não & $0(0)$ & $1(14,3)$ & $3(42,9)$ & $3(42,9)$ & & $2(28,6)$ & $2(28,6)$ & $3(42,9)$ & $0(0)$ & \\
\hline Satisfeito método & & & & & 0.039 & & & & & 0.471 \\
\hline $\operatorname{Sim}$ & $30(30)$ & $20(20)$ & $36(36)$ & $14(14)$ & & $65(65)$ & $22(22)$ & $11(11)$ & $2(2)$ & \\
\hline Não & $1(3,6)$ & $9(32,1)$ & $13(46,4)$ & $5(17,9)$ & & $15(53,6)$ & $10(35,7)$ & $2(7,1)$ & $1(3,6)$ & \\
\hline
\end{tabular}

* Qui quadrado de Pearson. Fonte: Autores (2021). 
Ao considerar a satisfação com a vida entre os que frequentavam ou não a sala de cuidados, foi identificado que aqueles que estavam dentro da sala apresentaram associação significativa com o grupo etário $(\mathrm{p}<0,004)$, satisfação com o curso ( $\mathrm{p}<0,050)$ e satisfação com o método $(p<0,015)$. Já os que estavam fora da sala de cuidados apresentaram associação com módulo perdido $(p<0,001)$ e satisfação com o curso $(p<0,038)$. Foi identificado que existe dependência condicional e que a relação da satisfação com a vida entre idade, satisfação com o curso e satisfação com o método é influenciada pelas práticas integrativas pelos estudantes (Tabela 03).

Tabela 3 - Características descritivas e associação de Pearson dos estudantes da área da saúde considerando a satisfação com a vida dentro e fora da sala de cuidados integrativos, Lagarto, Sergipe, 2020.

\begin{tabular}{|c|c|c|c|c|c|c|c|}
\hline \multirow{3}{*}{$\begin{array}{c}\text { Características } \\
\text { sociodemográficas } \\
\text { e de currículo }\end{array}$} & \multicolumn{6}{|c|}{ Satisfação com a vida autodeclarada pelos estudantes } & \multirow[b]{3}{*}{ p-valor ${ }^{b}$} \\
\hline & \multicolumn{3}{|c|}{ Dentro da Sala de Cuidados } & \multicolumn{3}{|c|}{ Fora da Sala de Cuidados } & \\
\hline & $\begin{array}{c}\text { Sim } \\
\mathrm{n}(\%)\end{array}$ & $\begin{array}{c}\text { Não } \\
\text { n (\%) }\end{array}$ & p-valor ${ }^{\mathrm{a}}$ & $\begin{array}{c}\mathrm{Sim} \\
\mathrm{n}(\%)\end{array}$ & $\begin{array}{c}\text { Não } \\
\text { n (\%) }\end{array}$ & p-valor ${ }^{\mathrm{a}}$ & \\
\hline \multicolumn{8}{|l|}{ Idade } \\
\hline$<19$ anos & $41(77,4)$ & $12(22,6)$ & \multirow{3}{*}{0,004} & $57(72,2)$ & $22(27,8)$ & \multirow{3}{*}{0,242} & \multirow{3}{*}{$0,021 *$} \\
\hline 20 e 29 anos & $88(80,7)$ & $21(19,3)$ & & $28(58,3)$ & $20(41,7)$ & & \\
\hline$>30$ anos & $0(0)$ & $3(100)$ & & $2(50)$ & $2(50)$ & & \\
\hline \multicolumn{8}{|l|}{ Módulo perdido } \\
\hline Sim & $20(71,4)$ & $8(28,6)$ & 0,493 & $4(28,6)$ & $10(71,4)$ & $>0,001$ & 0,058 \\
\hline Não & $110(77,5)$ & $32(22,5)$ & & $84(70)$ & $36(30)$ & & \\
\hline \multicolumn{8}{|l|}{ Satisfeito com o curso } \\
\hline Sim & $118(79,2)$ & $31(20,8)$ & 0,050 & $84(68,3)$ & $39(31,7)$ & 0,038 & 0,011 \\
\hline Não & $10(58,8)$ & $7(41,2)$ & & $2(28,6)$ & $5(71,4)$ & & \\
\hline \multicolumn{8}{|l|}{ Satisfeito com o método } \\
\hline Sim & $109(80,1)$ & $27(19,9)$ & 0,015 & $69(69)$ & $31(31)$ & 0,403 & 0,040 \\
\hline Não & $18(60)$ & $12(40)$ & & $17(60,7)$ & $11(39,3)$ & & \\
\hline
\end{tabular}

a Qui quadrado de Pearson / b Teste de Mantel-Haenszel / * Modelo de Independência Condicional Log-linear. Fonte: Autores (2021).

A Tabela 4 expõe a razão de possibilidades entre o grupo de alunos que frequentaram ou não a sala de cuidados. Aqueles alunos que relataram estar insatisfeitos com a vida apresentam 2,6 vezes mais chances $(p<0,02)$ de não frequentarem a sala de cuidados integrativos. E os que declararam estar insatisfeitos com o curso apresentam 2,68 vezes mais chances ( $<<0,06)$ de não frequentarem a sala de cuidados. A relação com o grupo etário identificou que a faixa etária entre 20 e 29 anos apresentam 3.67 $(\mathrm{p}<0.001)$ vezes mais chances de não frequentarem a sala de cuidados quando comparado aqueles menores de 19 anos. 
Tabela 4 - Modelo de regressão logística múltipla considerando os estudantes de graduação que não frequentam a sala de cuidados integrativos.

\begin{tabular}{lll}
\hline \multicolumn{1}{c}{ Características dos estudantes } & ORa & p-valor (IC) \\
\hline Ansiedade & & \\
Sim & 1.36 & $0.35(0.71-2.59)$ \\
Não & & \\
Depressão & 1.24 & $0.45(0.69-2.22)$ \\
Sim & & \\
Não & $\mathbf{2 . 0 6}$ & $0.02(1.12-3.79)$ \\
Satisfação com a vida & & \\
Sim & 1.46 & $0.64(0.29-7.24)$ \\
Não & 0.39 & $0.25(0.08-1.95)$ \\
Grupo etário & & $<0.001$ \\
$<19$ anos & & \\
20 e 29 anos & $\mathbf{2 . 6 8}$ & $0.06(0.94-7.66)$ \\
$>30$ anos & & \\
Satisfeito com o curso & & \\
Sim & & \\
Não & & \\
\hline
\end{tabular}

ROC curve: $69 \%$; $<<0,001$; IC:0,63 - 0,75. Fonte: Autores (2021).

\section{Discussão}

A universidade pode funcionar como uma porta de entrada para a vida profissional, ou para alguns, o critério para obtenção/manutenção do emprego. Com isso, muitos estudantes possuem a necessidade da dupla jornada: a de trabalho e a acadêmica. (Freitas et al., 2018). Apesar de ser essa a realidade de muitos estudantes, em nosso estudo, a maioria dos estudantes não possuía trabalho formal assim como os estudantes da pesquisa realizada por Leão (2018) na qual a maioria também não exercia atividade remunerada bem como era solteira e vivia com a família.

Foi identificado uma heterogeneidade quanto aos níveis de ansiedade, na qual os níveis moderados foram os mais elevados considerando aqueles que participam ou não da sala de Cuidados Integrativos. Em contrapartida, Fernandes e colaboradores (2018) observaram mais casos de estudantes com grau mínimo e leve de ansiedade.

De acordo com Mesquita et al. (2016), é importante salientar que os estudantes podem enfrentar dificuldades de adaptação e atravessar mudanças em sua rotina que desenvolva transtornos mentais. A realidade acadêmica, a iniciação à atividade clínica, o contato com pacientes com doenças graves e terminais entre outros pode, ainda, contribuir para o surgimento de dificuldades interpessoais e o aparecimento de sintomas de sofrimento psíquico a outros fatores agravantes.

Diante deste cenário, observamos que os alunos que foram analisados dentro da sala apresentaram sintomas moderados de ansiedade (45.3\%) ainda assim, é importante destacar que quase 1/3 dos estudantes que procuraram as práticas integrativas já apresentavam sintomas discretos de depressão.

O sexo dos estudantes não apresentou associação positiva com os sintomas de ansiedade e depressão entre os frequentadores da sala de cuidados, entretanto um fator interessante para se abordar é que mais da metade desses estudantes é do sexo feminino. Para Andrade e Pires (2020), esses dados fortalecem a ideia que mulheres costumam buscar mais cuidados, sobretudo psicológicos, em relação aos homens.

O número de alunos com sintomas moderados de ansiedade esteve mais associado ao sexo feminino entre aqueles que frequentaram e entre aqueles que não frequentaram a sala de cuidados. O fato justifica-se ao compreender que é maior a prevalência da ansiedade em mulheres (Gama et al., 2008) por causa de sua necessidade em superar mais obstáculos impostos 
pela sociedade que ainda é muito machista como afirma Borine (2015) quando busca compreender a relação entre qualidade de vida e estresse em acadêmicos da área da saúde.

Nesta pesquisa, a maioria dos alunos já tinha cursado mais de $50 \%$ do curso entre os frequentadores e não frequentadores da sala de cuidados. Apesar da associação positiva encontrada entre o período cursado e sintomas de ansiedade entre os frequentadores da sala de cuidados, Lelis (2020) e colaboradores identificaram que o período que o estudante se encontra durante o ambiente universitário não apresenta relação com a prevalência de ansiedade.

Existem diversas queixas que motivam o estudante a procurar as práticas integrativas e complementares. A ansiedade foi a mais recorrente considerando o campus do interior de Sergipe bem como no estudo realizado na Universidade Federal do Sul da Bahia (Belasco et al., 2019).

No tocante à condição de viver com a família, foi encontrada relação positiva com sintomas de ansiedade entre os alunos que não frequentaram a sala de cuidados. Por outro lado, não houve associação entre aqueles que frequentaram a sala de cuidados. Nesse sentido, a literatura identificou que existe relação significativa entre o nível de ansiedade e com quem o estudante reside, sendo que quem mora com pais ou familiares tem níveis menores de ansiedade, além de ter qualidade de vida melhor (Vizzoto et al., 2017).

No ambiente acadêmico, a prevalência de ansiedade é significativamente grande independente dos níveis que a ansiedade apresente (Bento et al., 2017) como corrobora o presente estudo. Assim, ratifica-se a necessidade de utilizar instrumentos e estratégias para a promoção da saúde mental dos estudantes universitários como sinaliza Souza (2017) sobre a ampliação das ofertas terapêuticas nos sistemas de saúde, que extrapolem o modelo biomédico.

Nesse sentido, as Práticas Integrativas e Complementares podem ampliar as contribuições apontadas pela medicina tradicional e auxiliar os estudantes universitários a atravessaram a experiência acadêmica que pode acarretar sofrimento mental. Belasco e colaboradores (2019) afirmam que as Universidades precisam imprimir os esforços para incluir em seu delineamento projetos transdisciplinares, não só no âmbito do ensino, mas em todos os aspectos da vida universitária.

Dessa forma, faz-se mister refletir sobre a necessidade de discutir a instrumentalização, divulgação, acesso e maior aprofundamento teórico dentro do ambiente acadêmico sobre as práticas integrativas e complementares em saúde. O campus de Lagarto oferece Práticas Integrativas aos seus alunos no Estado por meio de uma parceria junto ao MOPS. Acreditamos que essa oferta é uma importante estratégia para promoção da saúde mental desses estudantes somada ao fato de que o acesso é facilitado geograficamente uma vez que a sala de cuidados se encontra dentro deste campus.

O pensamento amplo ao considerar ansiedade e depressão entre os universitários também fomenta a necessidade de discutir sobre o bem-estar subjetivo (Medeiros \& Martins, 2020) e suas formas de entendimento. Uma delas, a satisfação com a vida se apresenta dentro do constructo do bem-estar e pode envolver fielmente a qualidade de vida dos estudantes nas suas próprias perspectivas. Assim como no estudo realizado por Bardagi e Hutz (2010), os escores deste estudo para a satisfação de vida se aproximam de outras investigações com estudantes universitários ao indicarem um percentual maior de alunos satisfeitos com suas condições de vida.

Nossos achados identificaram que a idade, perda de módulo, satisfação com o curso e com o método, estiveram associadas com satisfação de vida em Sergipe. As poucas pesquisas nacionais que investigam satisfação de vida em universitários conservam-se, em sua maioria, na qualidade/satisfação de vida em alunos de uma área ou curso específico (Bardagi \& Hutz, 2010).

A idade e satisfação com a vida desses estudantes mostrou associação positiva, todavia, no estudo publicado por Lima e Moraes (2016) que traz como público-alvo adolescentes, tal correlação apresentou-se negativa. Nesse sentido, segundo Pachane (2004), os aspectos relacionados à satisfação costumam ser os relacionamentos pessoais, a aprendizagem, o crescimento pessoal, 
a formação profissional e a qualidade do curso e da universidade.

Concernente ao grupo etário, a idade apresentou relação positiva considerando a participação ou não dentro da sala de cuidados. A relação com o grupo etário identificou que a faixa etária entre 20 e 29 anos apresentam 3.67 vezes mais chances de não frequentarem a sala de cuidados quando comparado aqueles menores de 19 anos.

Os alunos que relataram estar insatisfeitos com a vida apresentam 2,6 vezes mais chances de não frequentarem a sala de cuidados. E os que declararam estar insatisfeitos com o curso apresentam 2,68 vezes mais chances de não frequentarem a sala de cuidados. Assim, sugere-se que o grau de insatisfação com a vida pode gerar impacto negativo na busca pelo cuidado em saúde física e mental, consequentemente menos motivados a buscarem novas práticas de cuidados em saúde e movidos, também, pela falta de conhecimento das práticas ofertadas e/ou preconceito. Nesse sentido, acreditamos que é possível que estes alunos não possuam conhecimentos suficientes acerca dos benefícios das práticas integrativas e complementares em saúde.

Como indicações de novos estudos, então, é preciso repensar a possibilidade de conhecer o grau de satisfação com a vida desses estudantes após o ingresso na Universidade e antes da conclusão do curso e ainda, fomentar a disseminação do conhecimento acerca das práticas integrativas e complementares no ambiente acadêmico e seus benefícios. Sugere-se então, novos estudos que possam mensurar as contribuições de práticas integrativas junto aos aspectos psíquicos do estudante universitário que poderão resultar em desdobramentos para pesquisas que ampliem o olhar para essa população.

\section{Conclusão}

O perfil dos alunos mostrou que o grupo etário mais prevalente possuía idade entre 20 e 29 anos, a maioria do sexo feminino, solteiro(a), sem trabalho formal, vivia com a família, não havia concluído mais de $50 \%$ da graduação e relatou satisfação com o curso e com o método de ensino adotado pela Universidade.

Entre aqueles que buscaram a sala, associação significativa foi encontrada entre os sintomas de ansiedade e o período em que os alunos se encontram na grade acadêmica, módulo perdido e queixa principal. Os sintomas de depressão apresentaram associação com o grupo etário, módulo perdido e satisfação com o curso.

Foi identificado que existe dependência condicional e que a relação da satisfação com a vida entre idade, curso, satisfação com o curso e satisfação com o método é influenciada pela condição de frequentar ou não a sala de cuidados. Entendemos que o grau de satisfação com o curso, com a vida bem como o grupo etário pode influenciar o grau de motivação pela busca da sala de cuidados. Conclui-se que há necessidade de melhor avaliação acerca dos fatores que podem motivar o comportamento exploratório pelas Práticas Integrativas.

\section{Referências}

Andrade, A. M., \& Pires, E. U. (2020). Avaliação dos níveis de ansiedade dos estudantes da UFRRJ. Revista Trabalho (En)Cena, 5(1): 248-468.

Bardagi, M., \& Hutz, C. (2010). Satisfação de vida, comprometimento com a carreira e exploração vocacional em estudantes universitários. Arquivos Brasileiros de Psicologia, 62, 159-170.

Belasco, I. C., Passinho, R. S., \& Vieira, V. A. (2019). Práticas integrativas e complementares na saúde mental do estudante universitário. Arquivos Brasileiros de Psicologia, 71, 103-111.

Bento, L. M. A., et al. (2017). Percepção dos alunos de medicina quanto a aprendizagem X ansiedade na metodologia ativa. Revista de Ensino, Educação e Ciências Humanas, 18(2): 178-182.

Borine, R. C. C., et al. (2015). Relação entre qualidade de vida e o estresse em acadêmicos da área da saúde. Estudos Interdisciplinares em Psicologia, 6(1): $100-118$.

Botega, N. J., et al. (1995). Transtornos do humor em enfermaria de clínica médica e validação de escala de medida (HAD) de ansiedade e depressão. Revista de saúde pública, 29, 359-363. 
Brasil. Ministério da Saúde. Secretaria de Atenção à Saúde. Departamento de Atenção Básica. Manual de implantação de serviços de práticas integrativas e complementares no SUS / Ministério da Saúde, Secretaria de Atenção à Saúde, Departamento de Atenção Básica. - Brasília: Ministério da Saúde, 2018.

Carleto, C. T. et al. (2018). Adaptação à universidade e transtornos mentais comuns em graduandos de enfermagem. Revista Eletrônica de Enfermagem, v. 20.

Cestari, V. R. F., et al. (2017). Estresse em estudantes de enfermagem: estudo sobre vulnerabilidades sociodemográficas e acadêmicas. Acta Paulista de Enfermagem, 30, 190-196.

Costa, D. S., et al. Sintomas de depressão, ansiedade e estresse em estudantes de medicina e estratégias institucionais de enfrentamento, 2020, 44(1): e040.

Depression, WHO. (2017). Other Common Mental Disorders: Global Health Estimates. World Health Organization, p. 1-24.

Diener, E. D., et al. (1985). The satisfaction with life scale. Journal of personality assessment, 49, 71-75.

Fernandes, M. A., et al. (2018). Prevalence of anxious and depressive symptoms in college students of a public institution. Revista brasileira de enfermagem, $71,2169-2175$.

Freitas, A. C. M. et. al. (2018). Fatores intervenientes na qualidade de vida do estudante de enfermagem. Rev. enferm. UFPE, $12,2376-2385$.

Gama, M. M. A. et al. (2008). Ansiedade-traço em estudantes universitários de Aracaju (SE). Revista de Psiquiatria do Rio Grande do Sul, $30,19-24$.

Hutz, C. S., Zanon, C., \& Bardagi, M. P. (2014). Satisfação de vida. Avaliação em psicologia positiva, p. 43-48.

Kumar, H., Shaheen, A., Rasool, I., \& Shafi, M. (2016). Psychological Distress and Life Satisfaction among University Students. J Psychol Clin Psychiatry, 5, 00283 .

Leão, A. M., et al. (2018). Prevalência e Fatores associados à Depressão e Ansiedade entre Estudantes Universitários da Área da Saúde de um Grande Centro Urbano do Nordeste do Brasil. Revista Brasileira de Educação Médica, 42, 55-65.

Lelis, K. C., et al. (2020). Sintomas de depressão, ansiedade e uso de medicamentos em universitários. Revista Portuguesa de Enfermagem de Saúde Mental, (23): 9-14.

Medeiros, L. K. A., \& Martins, R. R. (2020). Análise Comparativa de Estudos sobre Bem-Estar Subjetivo no Brasil: Aspectos Epistemológicos, Metodológicos e Teóricos. Estudos e Pesquisas em Psicologia, 20, 953-975.

Mesquita, A. M., et al. (2016). Depressão entre estudantes de cursos da área da saúde de uma universidade em Mato Grosso. Journal Health NPEPS, 1

Pachane, G. G. (2004). A experiência universitária e sua contribuição ao desenvolvimento pessoal do aluno. In: Mercuri, E., Polydoro, S. A. J. (Ed.). Estudante universitário: características e experiências de formação. Taubaté, São Paulo: Cabral Ed. e Livraria Universitária, p.155-186.

Santos, L. L., de Miranda, S. P., Clemente, V. C., \& Nogueira, M. C. (2018). Conhecimento e aceitação das práticas integrativas e complementares por estudantes de medicina. Revista de APS, 21(4).

Silva, E. C., \& Heleno, M. G. V. (2012). Qualidade de vida e bem-estar subjetivo de estudantes universitários. Revista Psicologia e Saúde.

Sousa, I. M. C., et al. (2012). Práticas integrativas e complementares: oferta e produção de atendimentos no SUS e em municípios selecionados. Cadernos de Saúde Pública, 28, 2143-2154.

Sousa, I. M. C., de Aquino, C. M. F., \& Bezerra, A. F. B. (2017). Custo-efetividade em práticas integrativas e complementares: diferentes paradigmas. JMPHC Journal of Management \& Primary Health Care| 8, 343-350.

Sousa, L. A., \& Barros, N. F. (2018). Integrative and Complementary Practices in the Unified Health System: progresses and challenges. Revista latinoamericana de enfermagem, v. 26.

Souto, E. P., et al. (2018). Percepção da disponibilidade do capital social e sua associação com os transtornos mentais comuns e depressão: resultados do ELSABrasil. Tese de Doutorado.

Suehiro, A. C. B., \& Andrade, K. S. d. (2018). Satisfação com a experiência acadêmica: um estudo com universitários do primeiro ano. Psicologia em Pesquisa, $12(2), 77-86$.

Vasconcelos T. C., et al. (2015). Prevalência de sintomas de ansiedade e depressão em estudantes de medicina. Revista Brasileira de Educação Médica, 39, p. $135-142$.

Vizzotto, M., et al. (2017). Saudades de casa: indicativos de depressão, ansiedade, qualidade de vida de estudantes universitários. Revista Psicologia e Saúde, 9(1): 59-73.

Zigmond, A. S, \& Snaith, R. P. (1983). The hospital anxiety and depression scale. Acta psychiatrica scandinavica, 67, 361-370. 